Title: Real-time Performance of Control Allocation for Actuator Coordination in Heavy Vehicles

Publisher: IEEE

DOI: $10.1109 /$ IVS.2009.5164359

Copyright: ( 2009 IEEE. Personal use of this material is permitted. Permission from IEEE must be obtained for all other uses, in any current or future media, including reprinting/republishing this material for advertising or promotional purposes, creating new collective works, for resale or redistribution to servers or lists, or reuse of any copyrighted component of this work in other works.

Version: Accepted article

To cite this article: TAGESSON, Kristoffer, et al. Real-time performance of control allocation for actuator coordination in heavy vehicles. In: Intelligent Vehicles Symposium, 2009 IEEE. IEEE, 2009. p. 685-690.

To link to this article: http://dx.doi.org/10.1109/IVS.2009.5164359 


\title{
Real-time Performance of Control Allocation for Actuator Coordination in Heavy Vehicles
}

\author{
Kristoffer Tagesson, Peter Sundström, Leo Laine and Nicolas Dela
}

\begin{abstract}
This paper shows how real-time optimisation for actuator coordination, known as control allocation, can be a viable choice for heavy vehicle motion control systems. For this purpose, a basic stability control system implementing the method is presented. The real-time performance of two different control allocation solvers is evaluated and the use of dynamic weighting is analysed. Results show that sufficient vehicle stability can be achieved when using control allocation for actuator coordination in heavy vehicle stability control. Furthermore, real-time simulations indicate that the optimisation can be performed with the computational capacity of today's standard electronic control units.
\end{abstract}

Index Terms-Real time systems, Road vehicle control, Algorithms, Optimization methods, Actuators, Safety

\section{INTRODUCTION}

Functional structure is important when designing vehicle motion control systems. Ideally, function design should conform to, rather than define, the control structure. In Fig. 1, a suggestion for a hierarchical control structure is shown. Starting at the top of the figure, the driver inputs are interpreted to determine what vehicle behaviour is desired by the driver. Driver support functions are then applied such as cruise control and lane keeping aid. The global forces required to achieve the desired motion are calculated in the Control Law block. Here, stability control is applied to prevent unstable vehicle behaviour by requesting correcting global forces. Finally, actuators are coordinated to deliver these global forces. With a high number of actuators in general this involves a non-unique solution. Furthermore, there is a supply of information to all functions in the hierarchy, in this case in the form of measured signals from the vehicle system. If this structure is maintained and the interface between the different functions is well-defined, each different function can be designed and tuned more or less separately, thus increasing reconfigurability.

By performing actuator coordination dynamically, the fault tolerance of the system can be increased. This since desired forces can be redistributed over still functioning actuators in the event of actuator failure. Furthermore, actuator usage can be adapted to physical circumstances such as tyre/road friction and also to actuator limitations by dynamically adjusting actuator priority and constraints. This dynamic coordination can be done using control allocation, a method originating

K. Tagesson, L. Laine and N. Dela are with the Department Chassis Strategies \& Vehicle Analysis, VOLVO 3P, Dept 26661, AB4S, SE-405 08 GÖTEBORG, Sweden, kristoffer.tagesson@volvo.com, leo.laine@volvo.com, nicolas.dela@volvo.com

P. Sundström is with MODELON AB, Ideon Science Park, SE-223 70 LUND, Sweden, peter.sundstrom@modelon. se

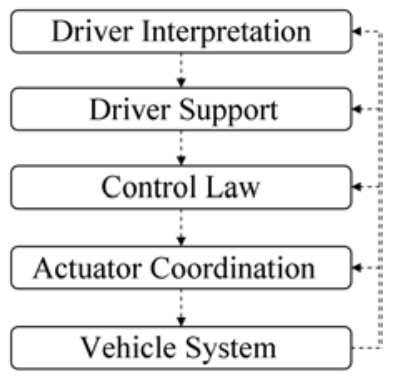

Fig. 1. Functional structure of a hierarchical motion control system. Arrows indicating flow of information.

from aviation and aerospace engineering. A main reference in the field is [1] where the method is thoroughly studied and developed for flight applications. Control allocation optimises actuator coordination, primarily with respect to global forces but also to desired actuator usage. When using the method, actuator coordination can be tuned separately which will allow the same control laws to be used for many different vehicle configurations without extensive tuning. As hybrid powertrains are introduced, the need for coordination between electric motors and mechanical brakes is introduced which can be effectively handled using control allocation, [2].

Control allocation for passenger cars has been researched for some time. In [2], [3] and [4] different applications of control allocation for vehicle motion control and energy management are adressed. In [5], control allocation is used for roll stability control of light trucks. The characteristics of two different control allocation solver techniques are studied in [6].

The real-time performance of the control allocation optimisation has yet to be fully evaluated. Furthermore, very little material is available on control allocation for motion control of heavy vehicles. In this paper, a stability control system for a solo truck with two rear axles is proposed to show that control allocation is a viable option for actuator distribution in motion control of heavy vehicles. The real-time performance of this system is studied to indicate whether the method can be implemented in production vehicles.

The outline of the paper is as follows: In section II the proposed control system is briefly presented along with the theory behind control allocation. Simulations performed are presented in section III and the corresponding results in section IV. Finally some conclusions are given in section V. 


\section{CONTROL SYSTEM}

When using the hierarchical functional structure, motion control system design can be separated into three parts according to Fig. 2. The Driver Interpretation block will be presented first, followed by the Control Law block. The control allocation method used in the Actuator Coordination block will then be presented more thoroughly followed by a brief description of the two solver techniques used.

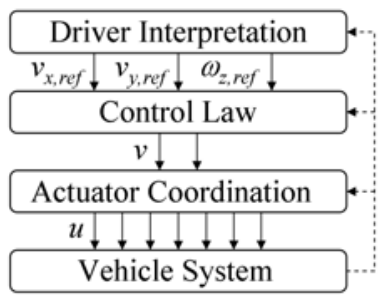

Fig. 2. Structure of the proposed control system. Actuator coordination is here performed using control allocation. Driver support functionality is excluded.

\section{A. Driver Interpretation}

The vehicle behaviour desired by the driver is generated by the Driver Interpretation block in Fig. 2 as the reference longitudinal speed, $v_{x, r e f}$, lateral speed, $v_{y, r e f}$, and yaw rate, $\omega_{z, \text { ref }}$. The desired longitudinal speed is calculated from pedal inputs. For the desired lateral behaviour of the vehicle, a reference model is used, in this case a linear one-track model, adapted from [7]. The driver is here assumed to expect a linear behaviour from the vehicle. With reference lateral speed, $v_{y, r e f}$, and yaw rate, $\omega_{z, r e f}$, as states and steering angle, $\delta$, as input, the state-space representation of the model is defined in Eq. (1). Here, the $A$-matrix is set dependent on the measured longitudinal speed of the real vehicle, $v_{x}$.

$$
\begin{gathered}
{\left[\begin{array}{c}
\dot{v}_{y, r e f} \\
\dot{\omega}_{z, r e f}
\end{array}\right]=\left[\begin{array}{cc}
\frac{1}{m} & 0 \\
0 & \frac{1}{I_{z z}}
\end{array}\right]\left(A\left[\begin{array}{c}
v_{y, r e f} \\
\omega_{z, r e f}
\end{array}\right]+\left[\begin{array}{c}
C_{\alpha 1} \\
L_{1} C_{\alpha 1}
\end{array}\right] \delta\right)} \\
A=\frac{1}{\left|v_{x}\right|}\left[\begin{array}{cc}
-\left(C_{\alpha 2}+C_{\alpha 1}\right) & C_{\alpha 2} L_{2}-C_{\alpha 1} L_{1}-m v_{x}^{2} \\
C_{\alpha 2} L_{2}-C_{\alpha 1} L_{1} & -\left(C_{\alpha 1} L_{1}^{2}+C_{\alpha 2} L_{2}^{2}\right)
\end{array}\right]
\end{gathered}
$$

where $C_{\alpha 1}$ and $C_{\alpha 2}$ are the sums of front and rear tyre cornering stiffnesses respectively. $L_{1}$ and $L_{2}$ are derived from the equivalent wheelbase, $L_{e}$. The vehicle configuration studied here is a three-axle truck with two rear axles. Due to the increased lateral stability of vehicles with multiple rear axles the equivalent wheelbase, [8], is used for the linear one-track model which is calculated as

$$
L_{e}=L\left(1+\frac{T}{L^{2}}\left(1+\frac{C_{\alpha 2}}{C_{\alpha 1}}\right)\right)
$$

where $L$ is the wheelbase of the real vehicle calculated as the distance from the front axle to the point around which the total torque generated by vertical loads of the rear axles add up to zero. $\mathrm{T}$ is the tandem factor which is calculated as

$$
T=\frac{\sum_{i=1}^{N} D_{i}^{2}}{N}
$$

where $\mathrm{N}$ is the number of rear axles and $D_{i}$ is the longitudinal distance from axle $i$ to the rear end of $L$. For determining $L_{1}$ and $L_{2}$, a term $\frac{T}{L^{2}} \frac{C_{\alpha 2}}{C_{\alpha 1}}$ is added to the distance from centre of gravity to the front axle to get $L_{1}$ and a term $\frac{T}{L^{2}}$ is added to the distance from the rear end of $L$ to centre of gravity to get $L_{2}$, [8].

To prevent the driver from requesting unrealisable behaviour from the vehicle, in the form of unattainable yaw rates, the yaw rate reference is limited to a maximum value, $\left|\omega_{z, \text { ref }}\right|_{\text {max }}$, based on tyre/road friction conditions. The maximum yaw rate reference is calculated as

$$
\left|\omega_{z, r e f}\right|_{\max }=\frac{\mu g}{v_{x}}
$$

where $\mu$ is the friction coefficient and $g$ is the gravitational acceleration.

\section{B. Control Law}

The Control Law block in Fig. 2 generates the virtual control vector, $v$, containing the global forces for controlling vehicle motion. The proposed stability control system includes yaw- and roll stability control. The yaw control system will be briefly presented here, for more details on the proposed yaw- and roll stability control system, see [9].

To detect yaw instability, the reference yaw rate, $\omega_{z, \text { ref }}$, from the linear reference model is compared to the measured vehicle yaw rate, $\omega_{z}$. When nonlinearities start to influence vehicle behaviour, primarily through saturation of tyre forces, the real vehicle behaviour will deviate from the linear reference indicating an unstable behaviour. A threshold, $\Delta_{\text {on }}$, is here introduced to prevent premature interventions. If the difference between reference yaw rate and measured yaw rate exceed this threshold, a correcting yaw torque is applied to prevent unstable behaviour. The control law is a proportional controller with the regulation error, $e_{\omega}$ defined as

$$
e_{\omega}=\left(\left|\omega_{z, r e f}-\omega_{z}\right|-\Delta_{o n}\right) \operatorname{sign}\left(\omega_{z, r e f}-\omega_{z}\right) .
$$

The correcting torque, $M_{z, T}$, is then calculated as

$$
M_{z, T}=K_{P} e_{\omega}
$$

where $K_{P}$ is the proportional control gain.

\section{Actuator Coordination}

In the general case a controlled system can often be approximated in the affine state space form

$$
\dot{x}=g(x)+h(x) u
$$

with $x$ as a vector including $k$ controlled states, $g, h$ as general functions and $u$ as the actuator input vector of size $m$. If a sufficiently short time interval is considered it further holds that $h(x) u \approx B u$, with $B \in \mathfrak{R}^{k \times m}$ known as the control 
effectiveness matrix. The problem concerned with control allocation is to achieve

$$
\left\{v=B u \mid u_{\min } \leq u \leq u_{\max }\right\}
$$

with $v$ as the virtual control vector, in this case formed by the global forces as $v=\left[F_{x, T}, M_{z, T}\right]^{T}$ with $F_{x, T}$ and $M_{z, T}$ representing the total requested longitudinal force and yaw torque respectively. Here $u_{\min }$ and $u_{\max }$ are actuator limits which are set not only from position limits but also from rate limits and what is physically realisable with tyres, e.g. brake action is limited by adhesive friction.

In general there is no guarantee for an existing $u$ to fulfil Eq. (9). In the case where there is no solution some arbitration rule has to be applied. On the other hand if the solution is not unique as $\operatorname{dim}(v)<\operatorname{dim}(u)$, see Fig. 2, some secondary rule has to be introduced, to achieve uniqueness. By using a weighted $\ell_{2}$-norm for both these rules the Weighted Least Squares problem for control allocation can be defined as

$$
u=\arg \min _{u_{\min } \leq u \leq u_{\max }}\left\|W_{u}\left(u-u_{d}\right)\right\|_{2}^{2}+\gamma\left\|W_{v}(B u-v)\right\|_{2}^{2}
$$

where $W_{u}, W_{v}$ are diagonal weighting matrices, $u_{d}$ the desired actuator input and $\gamma$ is a scalar usually set high to emphasise the control error. The uniqueness of (10) is guaranteed if $B$ has full rank, $\gamma>0$ and $W_{u}, W_{v}$ have positive diagonal elements [10].

Interesting parameters in Eq. (10), from a motion control perspective, are: $B$ - to achieve the correct global forces, $u_{d^{-}}$ to request a certain actuator usage and the weighing matrices $W_{u}$ and $W_{v^{-}}$to favour certain actuators and global forces respectively. For example, with a hybrid powertrain, $u_{d}$ can be used to request regeneration in electric motors with respect to energy buffer state of charge. Furthermore, $W_{v}$ can be varied with friction to prioritise either yaw- or roll stability control, since these two often counteract, [9]. The selection of $B$ and $W_{u}$ will here be further discussed.

1) Control Effectiveness matrix: The contribution of each actuator to the global forces is described by the control effectiveness matrix, B. This matrix should be set with respect to vehicle properties and actuator configuration. For the studied application, a truck model with 6 wheels and individual mechanical brakes was used. In this case a simple model of the longitudinal force induced from brake pressure is $F_{x i}=k_{c} \cos \delta_{i} P_{i}$, with $k_{c}$ as the proportional relation between brake pressure and tyre force, $\delta_{i}$ as the steering angle and $P_{i}$ as the brake pressure of tyre $i$. Analogously for lateral forces, $F_{y i}=k_{c} \sin \delta_{i} P_{i}$. Small steering angles are assumed and therefore the lateral forces achieved from braking are neglected and $\cos \delta_{i} \approx 1$ is used. The sum of the individual longitudinal forces form the total longitudinal force, $F_{x, T}$.

The resulting torque, $M_{z, T}$, on the vehicle around centre of gravity is calculated as the longitudinal force at each wheel multiplied by the corresponding lever arm. Together with the longitudinal force, this makes $B u$ equal to

$$
\underbrace{\left[\begin{array}{cccccc}
-k_{c} & -k_{c} & -k_{c} & -k_{c} & -k_{c} & -k_{c} \\
\frac{k_{c} b_{f}}{2} & -\frac{k_{c} b_{f}}{2} & \frac{k_{c} b_{m}}{2}-\frac{k_{c} b_{m}}{2} & \frac{k_{c} b_{r}}{2} & -\frac{k_{c} b_{r}}{2}
\end{array}\right]}_{B} \underbrace{\left[\begin{array}{c}
P_{f l} \\
P_{f r} \\
P_{m l} \\
P_{m r} \\
P_{r l} \\
P_{r r}
\end{array}\right]}_{u}
$$

with $b_{f}, b_{m}, b_{r}$ as front, middle and rear track width respectively. In the vector $u$, subindices denote front left, ..., rear right.

2) Dynamic Weighting $W_{u}$ : For braking of road vehicles, slip control is often used to maintain equal slip on all tyres. If tyre properties are assumed equal, this can be achieved by setting brake action in proportion to tyre vertical load. Since no yaw torque is desired from pure braking, the brake distribution should be equal for two tyres on the same axle. If tyres start to approach their limit in force capacity, brake distribution should be adjusted to avoid rear axle wheel lock resulting in oversteer. The notation $F_{z i}$, with $i \in\{f l, f r, m l, m r, r l, r r\}$, is here used as axle vertical load for the axle corresponding to tyre $i$, i.e. $F_{z f l}=F_{z f r}$. Given a measurement or good estimate of $F_{z i}$ brake action can be set in proportion to axle vertical load by setting $W_{u}$ dynamically as

$$
W_{u}=\sqrt{m g} \operatorname{diag}\left[\frac{1}{\sqrt{F_{z f l}}} \frac{1}{\sqrt{F_{z f r}}} \frac{1}{\sqrt{F_{z m l}}} \frac{1}{\sqrt{F_{z m r}}} \frac{1}{\sqrt{F_{z r l}}} \frac{1}{\sqrt{F_{z r r}}}\right]
$$

where $m$ is vehicle mass and $g$ is gravitational acceleration.

Assuming that the desired deceleration is achievable without saturating tyre forces, left and right side symmetry makes the achieved yaw torque equal to zero as long as $M_{z, T}=0$. The last term in Eq. (10) can then be set to zero, that is $B u=v$. With $u_{d}=[0, \ldots, 0]^{T}$ the remaining problem is

$$
u=\arg \min _{\text {subj. } \sum_{i} F_{x i}=F_{x, T}}\left\|W_{u} u\right\|_{2}^{2}
$$

where $u_{i}$ denotes braking pressure and $F_{x i}$ the longitudinal force, attained at wheel $i \in\{f l, f r, m l, m r, r l, r r\}$, from this brake pressure. It is here assumed that $u_{i}$ and $F_{x i}$ are proportionally related, i.e. $F_{x}=k_{c} u$. By using (12) this problem can be rewritten as

$$
F_{x}=\arg \min _{s u b j . \sum_{i} F_{x i}=F_{x, T}} \sum_{i} \frac{F_{x i}^{2}}{F_{z i}} .
$$

The Lagrangian function relating to this problem is

$$
\Lambda=\sum_{i} \frac{F_{x i}^{2}}{F_{z i}}-\lambda\left(\sum_{i} F_{x i}-F_{x, T}\right)
$$

where $\lambda$ is the Lagrangian multiplier. The optimal solution of (14) coincide with the minimisation of (15), [10], given by

$$
\frac{\partial \Lambda}{\partial \lambda}=\frac{\partial \Lambda}{\partial F_{x i}}=0
$$


This gives

$$
\left\{\begin{array}{l}
F_{x, T}=\sum_{i} F_{x i} \\
2 \frac{F_{x i}}{F_{z i}}=\lambda
\end{array}\right.
$$

By summing over $i$ for the last property and then use the first property the solution is seen to be

$$
F_{x i}=\frac{F_{z i}}{F_{z, T}} F_{x, T}=\frac{F_{z i}}{m g} F_{x, T}
$$

with $F_{z, T}=\sum_{i} F_{z i}=m g$.

As stated earlier, what is desired is brake action set in proportion to axle vertical load, $F_{z i}$. According to (18), this is exactly what is realised by setting the weighting matrix $W_{u}$ as in Eq. (12), given a good estimate of $F_{z i}$ and that unsaturated braking is performed. Furthermore, to maintain a well conditioned problem in Eq. (10) all $F_{z i}$ used in the calculations have to include a positive lower limit, to avoid division by zero.

\section{Control Allocation Solver Techniques}

In [1] a conventional Active Set method is suggested to be used for solving Eq. (10). A particular benefit from using this method in a control problem is that the solution from the previous sample can be used as an initial guess when optimising, known as warm-starting. This drastically reduces the mean number of iterations to convergence. In [6] a Primal-dual Interior Point (IP) method is suggested and compared to the Active Set method. The conclusion drawn is that the Primal-dual IP method would be more suitable when the number of actuators is high, that is around 20 and above while, for lower numbers, the two methods exhibit similar characteristics. In the following sections the basics of the two methods will be described, for further details see [1], [6], [9].

1) Active Set: With Active set Eq. (10) is first rewritten to match the standard least squares problem form as

$$
u=\arg \min _{u_{\min } \leq u \leq u_{\max }}\left\|\left[\begin{array}{c}
\gamma^{1 / 2} W_{v} B \\
W_{u}
\end{array}\right] u-\left[\begin{array}{c}
\gamma^{1 / 2} W_{v} v \\
W_{u} u_{d}
\end{array}\right]\right\|_{2}^{2}
$$

The approach here is to start at an initial guess of the solution. The elements in $u$ which are on the border of the feasible set are made fixated, other elements are left completely free. For these free elements the optimal solution, with respect to Eq. (19), is calculated. The attained solution is then checked for feasibility followed by optimality. If the solution is not feasible the associated element is made fixated. If a feasible solution is not optimal, one of the fixated elements is made free. The method continues in this manner until the optimum is reached. Convergence is fast in general, but the upper number of iterations can be high.
2) Primal-dual Interior Point: With Primal-dual IP Eq. (10) is first rewritten to match the standard quadratic form. By utilising the relation $\|\cdot\|^{2}=(\cdot)^{T}(\cdot)$ the standard form is

$$
\begin{aligned}
& \min _{x} \frac{1}{2} x^{T} H x+c^{T} x \\
& \text { subject to } x+w=x_{\text {max }}, \quad x \geq 0, \quad w \geq 0
\end{aligned}
$$

with

$$
\begin{aligned}
& H=2\left(B^{T} W_{v}^{T} W_{v} B+\frac{1}{\gamma} W_{u}^{T} W_{u}\right) \\
& c^{T}=-2\left(\left(v-B u_{\text {min }}\right)^{T} W_{v}^{T} W_{v} B+\frac{1}{\gamma}\left(u_{d}-u_{\text {min }}\right)^{T} W_{u}^{T} W_{u}\right) \\
& x=u-u_{\text {min }} \\
& x_{\text {max }}=u_{\text {max }}-u_{\text {min }}
\end{aligned}
$$

where $w$ is used as a slack-variable.

The method then utilises a barrier Lagrangian function as a dual problem. Included in this function is a parameter $\mu_{p d}$, known as the complementary gap, which together with the Karush-Kuhn-Tucker conditions defines the central path, leading the primal variables $x, w$ to the optimal solution. The equations for this central path are however nonlinear which is why a linearisation is performed. When $\mu_{p d}$ is calculated as small enough the solution is considered close enough to the optimal solution.

One characteristic of the method is that it requires a fairly stable number of iterations to reach the final solution. Another is that the choice of starting point has little influence on rate of convergence, [11]. This choice is therefore used to simplify calculations instead of incorporating warm-starting. Furthermore, all iterations maintain a feasible solution, just as the Active Set method.

\section{SIMULATIONS}

The proposed control system has been evaluated with respect to vehicle stability control performance as well as real-time computational performance. A truck model with 6 individually braked wheels was used for all simulations. The model includes dynamics for longitudinal and lateral speed as well as yaw rate. Furthermore, vehicle roll- pitch- and warp dynamics, a nonlinear tyre model and actuator dynamics are included. For more details on the vehicle model see [9].

\section{A. Stability Control Performance}

The performance of the yaw control system was evaluated using a Sine with Dwell manoeuvre, [12]. It is designed to trigger an oversteer behaviour in road vehicles. The initial speed used was $v_{0}=50 \mathrm{~km} / \mathrm{h}$ and friction was set as $\mu=0.2$.

The steering wheel angle amplitude, $A_{S W A}$, was varied as $40^{\circ}-200^{\circ}$ with yaw control switched on and off. Control system parameters used were: Intervention threshold $\Delta_{o n}=$ 0.035 , yaw proportional gain $K_{p}=8 \cdot 10^{5}$, weighting matrix $W_{v}=\operatorname{diag}[0.5,10]$ and weighting matrix $W_{u}$ set as in section II-C2.

As a measurement of vehicle stability the following constraints were used: lateral displacement of vehicle $3.5 \mathrm{~s}$ 
after the start of the manoeuvre, should be $d y>2 \mathrm{~m}$ for $A_{S W A}>100^{\circ}$, maximum vehicle sideslip should be $\beta_{\max }=$ $\max \tan ^{-1}\left(v_{y} / v_{x}\right)<20^{\circ}$, the yaw rate as a proportion of the maximum yaw rate measured $2 \mathrm{~s}$ after completing the manoeuvre should be $\frac{\omega_{z}\left(t_{C O S}=2\right)}{\omega_{z, \max }}<0.35$ and the same measurement $3.5 \mathrm{~s}$ after completing the manoeuvre should be $\frac{\omega_{z}\left(t_{\operatorname{CoS}}=3.5\right)}{\omega_{z, \max }}<0.2$.

\section{B. Real-time Performance}

The performance of the two solver techniques were evaluated for varying numbers of actuators. This was done by running 1800 simulations with random inputs for each number of actuators between 7 and 100, for more details see [9].

The proposed control system was also implemented in a real-time environment to provide deterministic measurements of execution times in the system. The real-time system was based on LabVIEW software and implemented in a Capax $3 \mathrm{GHz}$ Real-Time computer. Execution times for the actuator coordination part of the system were monitored separately during a Sine with Dwell manoeuvre with $A_{S W A}=150^{\circ}$. This provided a measurement for the real-time performance of the two solver techniques. To be mentioned is that throttle was used for allocation in this test case in addition to mechanical brakes, that is there were a total of 7 actuators instead of 6 .

\section{RESULTS}

Results acquired from simulations are presented and discussed in this section.

\section{A. Stability Control Performance}

The uncontrolled system is stable up to $A_{S W A}=60^{\circ}$. At $A_{S W A}=80^{\circ}$ an uncontrolled spinout occurs. When yaw control is turned on, the system stays stable for all amplitudes up to $200^{\circ}$ with respect to all constraints. Results are presented in Table I.

TABLE I

RESULTS OF SINE WITH DWELL TEST CASE - YAW CONTROL ON AND OFF

\begin{tabular}{|c|c|c|c|c|c|}
\hline$A_{S W A}$ & $\underset{t=3.5}{d y}$ & $\frac{\omega_{z}\left(t_{\operatorname{CoS}}=2\right)}{\omega_{z, \max }}$ & $\frac{\omega_{z}\left(t_{\operatorname{CoS}}=3.5\right)}{\omega_{z, \max }}$ & $\beta_{\max }$ & $Y C$ \\
\hline$\left[{ }^{\circ}\right]$ & {$[\mathrm{m}]$} & - & - & {$\left[{ }^{o}\right]$} & - \\
\hline & $>2$ & $<0.35$ & $<0.2$ & $<20$ & - \\
\hline 40 & 1.95 & $\overline{0}$ & $\overline{0}$ & 1.0 & $\overline{\text { off }}$ \\
\hline 60 & 2.51 & 0 & 0 & 2.6 & off \\
\hline 80 & 2.84 & 0.84 & 0.85 & 33.9 & off \\
\hline 100 & 3.05 & 1 & 1 & $>90$ & off \\
\hline 60 & 2.52 & 0 & 0 & 2.6 & on \\
\hline 80 & 2.88 & 0.02 & 0 & 5.2 & on \\
\hline 100 & 3.10 & 0.17 & 0 & 7.8 & on \\
\hline 120 & 3.24 & 0.18 & 0.001 & 10.2 & on \\
\hline 140 & 3.35 & 0.18 & 0.004 & 10.7 & on \\
\hline 160 & 3.44 & 0.17 & 0.009 & 11.2 & on \\
\hline 180 & 3.50 & 0.17 & 0.020 & 11.7 & on \\
\hline 200 & 3.55 & 0.17 & 0.041 & 12.2 & on \\
\hline
\end{tabular}

Fig. 3 shows how the yaw control system intervenes during the manoeuvre with $A_{S W A}=120^{\circ}$. The top two plots show the left and right side brake pressures, the middle plot shows the activation of the yaw control system and the bottom plot shows the yaw rate reference with the intervention thresholds and the measured vehicle yaw rate. During this specific case there is both over- and understeer behaviour which is corrected by the control system.

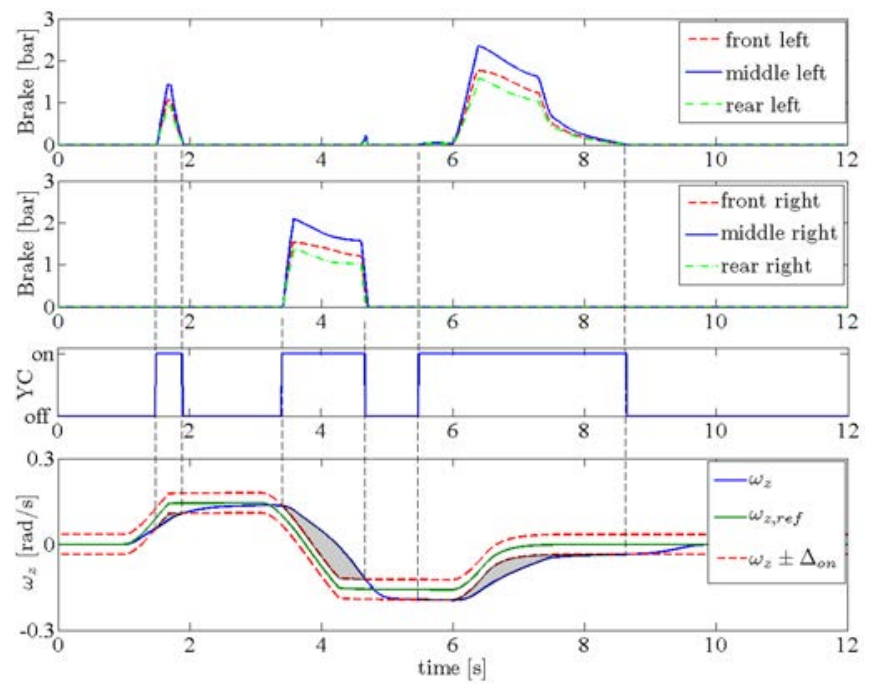

Fig. 3. Yaw control interventions, realised by individual left and right side brake actions. The manoeuvre performed is Sine with Dwell, $A_{S W A}=120^{\circ}$.

\section{B. Real-time Performance}

The characteristics of the two solver techniques when varying the number of actuators are shown in Fig. 4. Active Set is superior for lower numbers of actuators. When the number of actuators increase, Active Set requires more and more iterations to find the solution while Primal-dual IP stays stable at an average of about 15 iterations. The latter can therefore be said to be superior for higher numbers of actuators. These results are in agreement with [6].

When running the proposed control system in real time the Active Set method proves to be quite a bit faster than the Primal-dual IP method. Fig. 5 shows histograms for execution time and number of iterations for both methods. The Active Set method uses an average of 1.02 iterations and a maximum of 9 iterations. The Primal-dual IP method, on the other hand, uses an average of 11.33 iterations and a maximum of 16 iterations. Here, Active Set takes advantage of warm-starting. When studying execution time, Active Set uses an average of $48.50 \mu \mathrm{s}$ and a maximum of $95 \mu \mathrm{s}$ to find the optimal solution. Primal-dual IP uses an average of $174.13 \mu s$ and a maximum of $225 \mu \mathrm{s}$ for the optimisation. The standard deviation in execution time is $4.24 \mu s$ for Active Set and $10.45 \mu s$ for Primal-dual IP.

Assuming that a standard production electronic control unit (ECU) of today has a processor clock rate of about $100 \mathrm{MHz}$, the presented real-time results can be used to estimate what execution times to expect if the proposed control system was to be implemented in a production vehicle. Since 

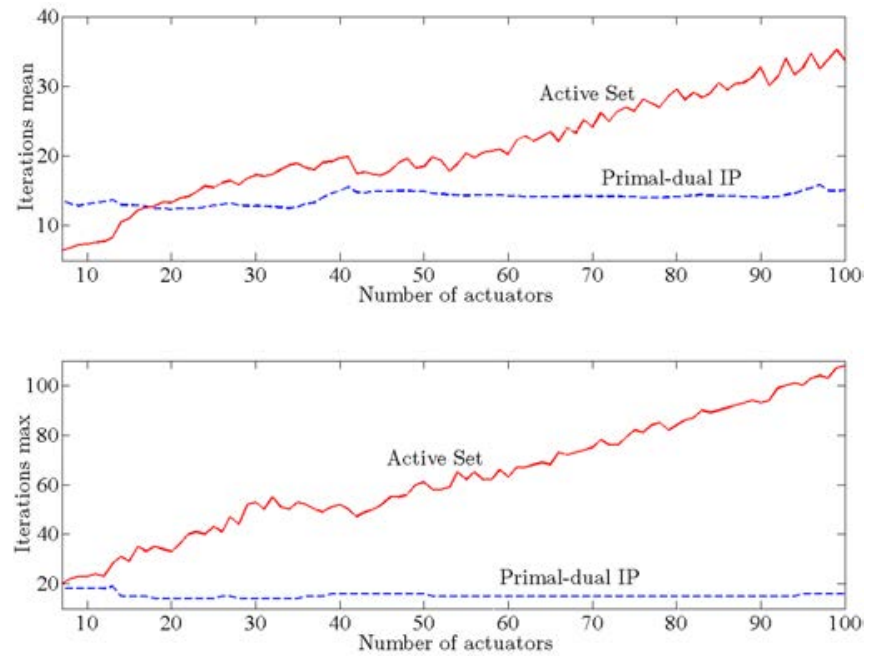

Fig. 4. Mean and maximum number of iterations for Active Set (Solid) and Primal-dual IP (dashed) when using random inputs and varying the number of actuators.
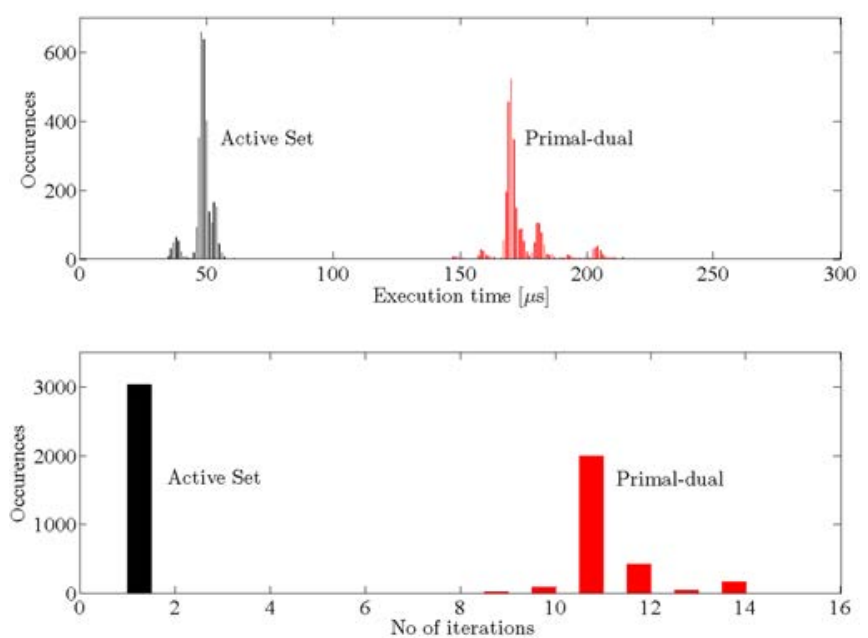

Fig. 5. Histograms of execution time (top) and number of iterations (bottom) for Active Set and Primal-dual IP when simulated in the real time environment. The first and last 100 iterations are removed to disregard long startup and termination iterations. The manoeuvre used is Sine with Dwell, $A_{S W A}=150^{\circ}$

the real-time computer has a clock rate of $3 \mathrm{Ghz}$ the realtime performance will be somewhere around 30 times slower if implemented in a standard production ECU. That means that the maximum execution time when using the Active Set method would be about $3 \mathrm{~ms}$. If using the Primal-dual IP method the corresponding value would be about $7 \mathrm{~ms}$. Since the sample time used in the control system was $10 \mathrm{~ms}$ these results indicate that the method is implementable in regular ECUs. It should however be noted that processor architecture and memory management, among other properties, differ substantially between the real-time computer and standard electronic control units. It is therefore important to see the results shown here as an indication, rather than proof, of sufficient performance.

\section{CONCLUSIONS}

The method of control allocation has been shown to be a viable alternative for actuator coordination in heavy vehicles. This was shown by implementing the method in a stability control system for a single unit truck which was evaluated with respect to both vehicle stability and computational performance.

The proposed vehicle stability control system prevents yaw instability for a Sine with Dwell manoeuvre when dynamically coordinating actuator usage by the means of control allocation. The acquired real-time performance figures indicate that the coordination can be performed within sample time requirements of the vehicle control system if implemented in a standard production ECU.

\section{ACKNOWLEDGMENTS}

The authors would like to thank Johan Sandqvist at Volvo 3P for assistance with the real-time implementation. Carsten Watolla at National Instruments is acknowledged for providing the LabVIEW software package used.

\section{REFERENCES}

[1] O. Härkegård, "Backstepping and control allocation with applications to flight control," Ph.D. dissertation, Department of Electrical Engineering, Linköping University, SE-581 83 Linköping, Sweden, May 2003.

[2] L. Laine, "Reconfigurable motion control systems for over-actuated road vehicles," Ph.D. dissertation, Department of Applied Mechanics, Chalmers University of Technology, SE-412 96 Göteborg, Sweden, June 2007.

[3] M. Jonasson, Aspects of Autonomous Corner Modules as an Enabler for New Vehicle Chassis Solutions. SE-100 44 Stockholm, Sweden: Licentiate thesis, Department of Vehicle Dynamics, Royal Institute of Technology, 2007.

[4] J. Andreasson, "On generic road vehicle motion modelling and control," Ph.D. dissertation, Department of Vehicle Dynamics, Royal Institute of Technology, Sweden, SE-100 44 Stockholm, Sweden, February 2007.

[5] B. Schofield, "Model-based vehicle dynamics control for active safety," Ph.D. dissertation, Department of Automatic Control, Lund University, SE-22100 Lund, Sweden, September 2008.

[6] J. A. M. Petersen and M. Bodson, "Constrained quadratic programming techniques for control allocation," IEEE TRANSACTIONS ON CONTROL SYSTEM TECHOLOGY, vol. 14, pp. 91-98, 2006.

[7] M. Klomp, On Drive Force Distribution and Road Vehicle Handling. SE-412 96 Göteborg, Sweden: Licentiate thesis, Department of Applied Mechanics, Chalmers University of Technology, 2007.

[8] C. B. Winkler, "Simplified analysis of the steady-state turning of complex vehicles," Vehicle System Dynamics, vol. 29, pp. 141-180, 1998.

[9] P. Sundström and K. Tagesson, "On real time adaptive and dynamically constrained control allocation for stability control of heavy vehicles," Master's thesis, Department of Signals and Systems, Chalmers University of Technology, SE-412 96 Göteborg, Sweden, December 2008.

[10] J. Nocedal and S. J. Wright, Numerical Optimization, 2nd ed. Springer, New York, USA, 2006.

[11] S. J. Wright, Primal-dual interior point methods. Siam, Philadelphia, USA, 1997

[12] L. Laine, S. Kharrazi, and N. Dela, "Proposal for using sine with dwell on low friction for the evaluation of yaw stability for heavy vehicle combinations," in IEEE International Conference on Vehicular Electronics and Safety, September 2008, pp. 163-167. 